\title{
Merger Driven Explosive Evolution of Distant Galaxies (Minor Mergers)
}

\author{
A.V. Kats ${ }^{1}$ and V.M. Kontorovich ${ }^{2,3}$ \\ ${ }^{1}$ Institute of Radio Physics and Electronics, National Academy of Sciences of \\ Ukraine, Kharkov, 61085, Ukraine; \\ ${ }^{2}$ Institute of Radio Astronomy, National Academy of Sciences of Ukraine, \\ Kharkov, 61002, Ukraine; \\ ${ }^{3}$ V.N.Karazin National University, Kharkov 61022, Ukraine; \\ vkont1001@yahoo.com
}

\begin{abstract}
We derived solutions for the Smoluchowski kinetic equation for the mass function of galaxies, which describes mergers in differential approximation, where mergers with low-mass galaxies are the dominant factor. The evolution of the initial distribution is analyzed as well as the influence of the source represented by galaxies (halos) that separate from the global cosmological expansion. It is shown that the evolution of the slope of the power-law part of the luminosity function at a constant mass-to-luminosity ratio observed in the Ultra Deep Hubble Field can be described as a result of explosive evolution driven by galaxy mergers. In this case the exponent depends exclusively on the uniformity degree of merger probability as a function of mass.
\end{abstract}

pacs: /, 95.30.Sf, 98.35.Ce, 98.62.Ck, 98.80.-k, 95.35.+d

keywords /, galaxy: minor merging, mass function, evolution

DOI: $10.1134 / \mathrm{S} 1990341313030012$

\section{INTRODUCTION}

In its generally adopted form the hierarchical clustering model yields selfsimilar solutions [1, 2, 3]. At the same time, investigations of distant galaxies reveal markedly non-self-similar behavior, which shows up, among other 
things, in the redshift dependence of the slope $\alpha$ of the power-law part of the Schechter luminosity function (LF) [4, 5, 6]

$$
\phi(L)=\phi_{*} L^{\alpha} \exp \left(-L / L_{*}\right) .
$$

(Here we do not discuss the parameters $\phi_{*}$ and $L_{*}$ of the Schechter function.) 1 We show that the observed evolution of the LF slope (Section 2), understood as the slope of the mass function (MF) of galaxies, can be described as a result of explosive evolution driven by galaxy mergers. Currently, mergers are believed to be the factor responsible for the evolution of types and masses of galaxies [7] (see also the discussion and references in the reviews [8, 9, 10]). Although the actual situation is more complex (see the recent reviews by Ellis and Silk [11] and Silk and Mamon [12]), we show that the observed parameters of the MF can be explained satisfactorily in terms of our hypothesis. We derive solutions for the Smoluchowski kinetic equation (KE) that describes merger-driven explosive evolution of the mass function of galaxies evolution of the mass function of galaxies [13, 14] in differential approximation [13, where mergers of massive galaxies with low-mass galaxies (minor mergers) play the main part (Sections 3 and 4). Note that the slope $\alpha$ of the MF of massive galaxies, which is proportional to the Schechter exponent $\propto M^{\alpha}$, can be written exclusively in terms of the uniformity degree $u$ of the merger probability as a function of mass (Sections 5 and 6). This property, in principle, makes it possible to determine the probability of mergers as a function of mass including that of the dark matter, based on observational data. In this paper we use the well-known dependencies of the probabilities of galaxy mergers on their mass to show that the observed evolution can be explained in terms of natural assumptions about merger mechanisms in different epochs (Sections 5 and 6). Explosive evolution occurs if the exponent $u>1$, which is evidently true for galaxy mergers. In the case of explosive evolution of an initial MF of sufficiently general form (i.e., decreasing faster than the square of the mass) a power-law asymptotic develops with the exponent $\alpha=-u$; in the case of predominant influence of a mass-localized source the exponent is equal to $\alpha=-(u+1) / 2$ (Section $6)$. Galaxies that separate from cosmological expansion play the role of the source. At large red shifts $z=6-8$ the exponent $u$ is determined by mergers

\footnotetext{
${ }^{1}$ At large masses the MF is not exponential, but decreases in accordance with a squareroot law (Section 7), which is due to the adopted model of source localization in the kinetic equation.
} 
of low-mass galaxies and is close to $u=2$. At small $z$, where more massive galaxies merge, gravitational focusing has to be taken into account [15] (see also Appendix 4). At $z \rightarrow 0$, when using a radius-mass relation of the form $R \propto \sqrt{M}$, which follows from the Tully-Fisher and Faber-Jackson relations 2 , this yields the well-known current value of the Schechter index $\alpha=-1.25$. At intermediate redshifts both the evolution of the MF driven by the source (in the domain of low masses) and the evolution of the initial mass distribution (in the domain of sufficiently large masses) result in the Schechter slope of $\alpha=-1.5$, which can be reconciled with observational data (Section 6 ). The above mechanisms of the formation of the MF (with the allowance for the assumed contribution of dark matter) alternate with each other in the process of evolution. Note that in the $u=2$ case we derived an exact solution of the kinetic equation with a local source in a wide mass range. In the general case of arbitrary $u>1$ we derived the asymptotic form of the solution of the kinetic equation at large masses on times close to the moment of "explosion." This result can qualitatively explain the observational data for the MF slope at the red shifts from $z=0$ to $z=8$.

The MF derived in the approximation considered (Section 7) has a form similar to that of the Schechter function, however, at large masses it decreases in accordance with a square-root law and not exponentially (see footnote 1). We determined the maximum mass (Section 7) cutoff of the MF as a function of time and parameters of the system in the vicinity of the "explosion" time. This approach also makes it possible, in principle, to determine the "explosion" times as a function of the initial conditions, interaction parameters, and merger mechanism (see Appendix). Our analysis is limited to the differential approximation and pairwise mergers (with the allowance for the dependence of merger probability on the masses of the galaxies involved). Despite these restrictions, the inferred MF slopes for explosive solutions agree satisfactorily with observations in a wide range of redshifts [16].

\section{OBSERVATIONS OF GALAXIES AT LARGE REDSHIFTS}

The advances in observations of galaxies at large $z$ are largely due to the use of multicolor photometry [17] in ultra deep fields of major telescopes and

\footnotetext{
${ }^{2}$ This exponent proved to be redshift dependent (see references in [11, 12]), but we do not take this fact into account in this paper.
} 
observations of distant galaxies through gravitational lenses. Let us mention, as a remarkable example, the observations of a possible galaxy merger at $z=2.9$ [18, 19].

The methods of multicolor photometry, which isolate the galaxies seen only through infrared filters because of the large redshifts,made it possible to construct galaxy luminosity functions out to $\mathrm{z}=8$ and even farther away based on decade-long observations of ultra deep fields on the Hubble Space Telescope and major ground-based telescopes. The Schechter LF slopes $\alpha$ (Fig. 1) determined in a series of papers by Bouwens, Illingworth, and their coauthors [4, 5], which we use below (see also numerous important references in [6, 11, 12, 16, 20] ), depend significantly on redshift. Note that the MF slope computed in hierarchical clustering models without the allowance for mergers [1, 2, 3] does not depend on z.

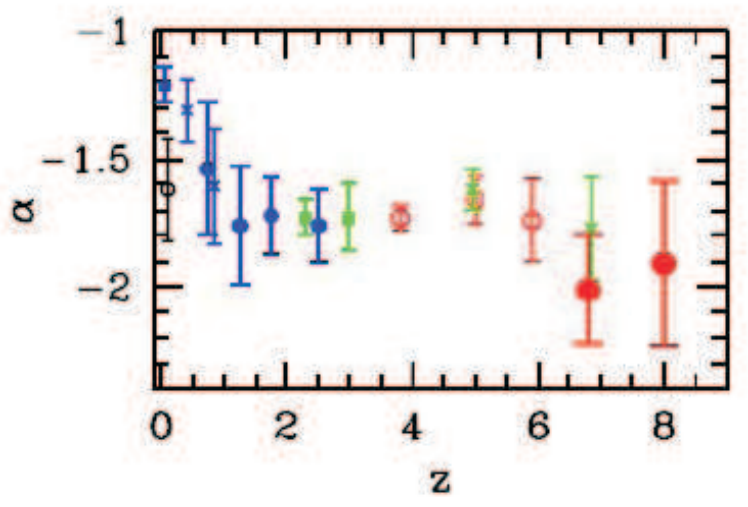

Figure 1: Variation of the Schechter slope with redshift in HUDF (the copy of Fig. 14 from [5] by courtesy of R.Bouwence).

\section{SMOLUCHOWSKI KINETIC EQUATION DESCRIBING MERGERS}

We now consider the differential version of the Smoluchowski kinetic equation for galaxy mergers with small mass increments [7]. It has the following form (see Appendix 1): 


$$
\frac{\partial}{\partial t} f(M, t)+C \Pi \frac{\partial}{\partial M}\left[M^{u} f(M, t)\right]=\varphi(M, t), \quad \Pi=\int_{0} d M_{2} M_{2} f\left(M_{2}, t\right) .
$$

Here we use the expression for the probability of galaxy mergers in the form $M^{u} / 2$ (see Appendix 2), where the exponent $u>1$. The $\Pi$ quantity is equal to the total mass of low-mass galaxies $\left(M_{2} \ll M\right)$, which is assumed to undergo no significant changes in the merger process (below we adopt $\Pi=$ Const). The presence of the source term $\varphi$ in the kinetic equation is essential; it describes the contribution of massive galaxies separating from cosmological expansion as gravitational instability develops [1, 2, 3].

Let us rewrite the kinetic equation in the form

$$
\begin{aligned}
& \frac{\partial}{\partial t} F(M, t)+C \Pi M^{u} \frac{\partial}{\partial M} F(M, t)=\Phi(M, t), \\
& F(M, t)=M^{u} f(M, t), \quad \Phi(M, t)=M^{u} \varphi(M, t)
\end{aligned}
$$

and use the method of characteristics to solve it. As a result, the kinetic equation reduces to the following set of ordinary differential equations

$$
\begin{aligned}
& d M / d t=C \Pi M^{u} \\
& d F / d t=\Phi
\end{aligned}
$$

The solution of the kinetic equation is an arbitrary function of independent first integrals of the equation set (4) [21]. We find this function based on the initial conditions (see Appendix 3). Integration of the first differential equation of equation set (4) yields

$$
\tau(t)+\frac{1}{(u-1) M^{u-1}}=a(M, t)=\text { const }, \quad \tau(t) \equiv C \int_{0}^{t} d t \Pi(t) \rightarrow C \Pi \cdot t .
$$

To integrate the second equation of the set, we first assume that the source $\varphi$ is local. More specifically, we assume that

$$
\Phi(M, t)=\delta(M-\bar{M}(t)) \Phi(t),
$$

where $\Phi(t)$ is a function of time whose form is of no importance to us. This assumption means that at time $t$ a galaxy of mass $\bar{M}(t)$ separates from cosmological expansion. We now write the mass $M$ in equation (6) in terms 
of the first integral a derived above, as $M=\mu(a, t)$, where, according to equation (5),

$$
\mu(a, t)=[(u-1)(a-\tau(t))]^{-\frac{1}{u-1}} .
$$

This allows us to find the second independent integral $b(M, t)$ of equation set (4) and construct the general solution of the kinetic equation.

\section{SOLUTION OF THE KINETIC EQUATION FOR A LOCALIZED SOURCE}

The kinetic equation (2) is linear, and therefore its solution has the form of a sum of two independent terms

$$
f(M, t)=f_{\text {in }}(M, t)+f_{s}(M, t) .
$$

The first term $f_{i n}$ can be written in terms of the initial distribution $f_{0}(M)$ (see Appendix) and contains only the integral a. The second term $f_{s}$ is associated with the source and contains the function $\bar{M}(t)$ that describes this source and monotonically increases with time 3 :

$$
\begin{aligned}
& f_{i n}(M, t)=\left[(u-1) \tau M^{u-1}+1\right]^{-\frac{u}{u-1}} f_{0}\left\{M\left[(u-1) \tau M^{u-1}+1\right]^{-\frac{1}{u-1}}\right\}, \\
& f_{s}(M, t)=M^{-u} K\left(\tau+\frac{1}{(u-1) M^{u-1}}, t\right)
\end{aligned}
$$

where

$$
\begin{aligned}
& K(a, t)=\int_{0}^{t} d x \delta[\mu(a, x)-\bar{M}(x)] \Phi(x)= \\
& \sum_{n} \Phi\left(x_{n}\right) \theta\left(t-x_{n}\right)\left|\frac{d}{d x}[\mu(a, x)-\bar{M}(x)]\right|_{x=x_{n}}^{-1} .
\end{aligned}
$$

Here $x_{n}$ - are the roots of equation (11)

$$
\mu(a, x)=\bar{M}(x),
$$

which determines the zero points of the $\delta$-function. It is important that $\bar{M}(t)$ is a monotonically increasing function of time [1, 2, 3]. For simplicity, we choose $\bar{M}(t)$ to be a linear function $M(t)=t / A$, and can therefore construct

\footnotetext{
${ }^{3}$ The explicit form of it, not playing the fundamental role, we specify below.
} 
for $u=2$ an exact "explosive" solution of the kinetic equation (see Appendix) and, for $u>1$, find the asymptotics in the domain of large masses near the "explosion" time $t_{c r}$ that are of interest to us. The explosive behavior of the solution 4 at $u>1$ found for galaxy mergers by Kontorovich, Kats and Krivitskii [13] and Cavaliere et al. [14] [14] means, as we shall see below, that an initially localized mass distribution formally reaches the domain of infinite masses in finite time (see [22, 23, 24] ).

\section{EXACT SOLUTION IN THE CASE OF QUADRATIC DEPENDENCE OF MERGER PROBABILITY ON MASS $(U=2)$ AND A SOURCE LINEAR IN TIME}

In this case, the equation for the roots of the arguments of the $\delta$-function (11) becomes quadratic $C \Pi \cdot x^{2}-a \cdot x+A=0$ with the following roots

$$
x_{ \pm}=\frac{a \pm \sqrt{a^{2}-a_{c r}^{2}}}{2 C \Pi}, a_{c r} \equiv 2 \sqrt{A C \Pi} .
$$

A real solution exists for $a \geq a_{c r}$, and the multiple root corresponds to the tangency of a hyperbola (into which the left-hand side of equation (11) turns at $u=2$, according to equation (7)) and a straight line (the right-hand side of the same equation, see Fig. 2). A multiple root (at time $t_{\text {tan }}$ of the tangency of the straight line and hyperbola) under the sign of the $\delta$-function is unacceptable5: at the critical value of parameter $a=a_{c r}$ the solution (10) becomes infinite. Below we overcome this restriction. At the same time, small differences $a-a_{c r}$ are of special importance to us, because they correspond to the case of sufficiently large masses and time t close to the "explosion" time $t_{c r}=2 t_{t a n}$ that we are interested in (the domain of large masses between the hyperbola $a=a_{c r}$ and the asymptote of this hyperbola, $T \equiv t / t_{t a n}=2$, see Fig. 2).

The contribution of the $\delta$-function of a complex argument $\delta(y(x))$ to the integral is known to be given by the following formula (the subscript numbers the roots of the argument) $\delta(y(x))=\sum_{i} \delta\left(x_{i}\right)\left|\frac{d y}{d x}\right|_{x_{i}}^{-1}$. If $y(x)$ is a quadratic

\footnotetext{
${ }^{4}$ The explosive behavior of the solutions of the Smoluchowski equation was first found by W. Stockmayer in 1943, and was then repeatedly rediscovered (see, e.g., references in reviews [21, 22, 23, 24]).

${ }^{5}$ To overcome this (model) restriction, we apply a very simple regularization in the next section by replacing the $\delta$-function with a step function of a small finite width.
} 


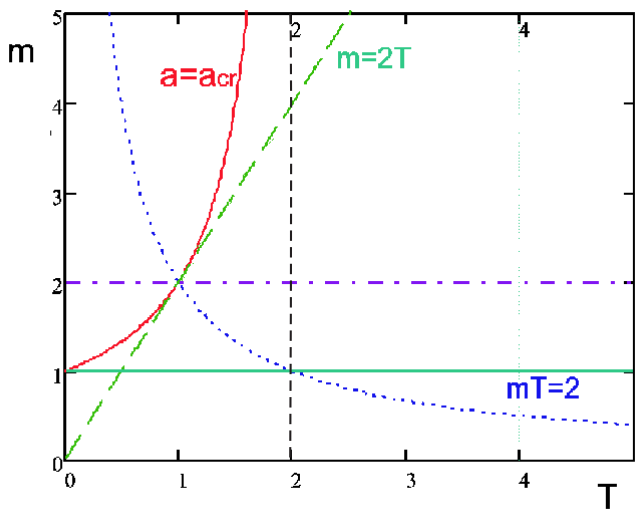

Figure 2: The domain of large masses $m \equiv a_{c r} M>2 T, 2 / T$ that is of interest to us lies between the hyperbola $a=a_{c r}$ and its vertical asymptote (the dashed line) $T \equiv t / t_{t a n}=2$, which corresponds to the time of the explosion, above the $m=2 T$ straight line. In this domain both roots of the $\delta$-function contribute to the MF.

trinomial, which we write in the form $y(x)=\left(x-x_{+}\right)\left(x-x_{-}\right)$, where $x_{ \pm}$are the roots of the equation $y(x)=0$, we derive, by virtue of $\frac{d y}{d x}=2 x-\left(x_{+}+x_{-}\right)$ the following two equations $\left.\frac{d y}{d x}\right|_{x=x_{+}}=x_{+}-x_{-} ;\left.\quad \frac{d y}{d x}\right|_{x=x_{-}}=-\left(x_{+}-x_{-}\right)$. It is evident that $\left|\frac{d y}{d x}\right|_{x=x_{ \pm}}|=| x_{+}-x_{-} \mid \propto \sqrt{a^{2}-a_{c r}^{2}}$. The non-vanishing factor under the sign of the $\delta$-function should not be differentiated, because the corresponding extra terms vanish when the roots are substituted. This simplifies the computations considerably. Thus the solution with a $\delta$-function source and a quadratic equation for its roots acquires the following form:

$$
K(a, t)=\frac{1}{\sqrt{a^{2}-a_{c r}^{2}}} \sum_{ \pm} \tilde{\Phi}\left(x_{ \pm}\right) \theta\left(a-a_{c r}\right) \theta\left(t-x_{ \pm}\right),
$$

where the tilde above $\Phi$ denotes the inclusion of constant multipliers that arise in the form of coefficients at the difference of the roots. We give the explicit formula in the Appendix. For small $a-a_{c r}$ only this difference remains under the square root sign in the denominator,

$$
K(a, t) \propto \frac{1}{\sqrt{a-a_{c r}}} .
$$


Below we will make sure that in the general case the asymptotics for the solution at large masses near the explosion time has a similar structure.

\section{POWER-LAW ASYMPTOTICS AT LARGE MASSES}

The above solution of the kinetic equation (9-11) can be used to find the power-law part of the distribution of galaxy masses, i.e., the exponent of the Schechter function at large masses in the vicinity of the "explosion" time $t=t_{c r}$, under the assumption of constant mass-to-luminosity ratio. Note that, as is evident from the part of solution $f_{\text {in }}(9)$ that is determined by the initial MF, the solution has the following asymptotics at large masses (see Appendix 3)

$$
f_{\text {in }} \propto M^{-u} \text {. }
$$

The physical meaning of this part of the solution is evident: it is a Kolmogorovtype distribution corresponding to a constant flow of the number of massive galaxies 6 along the mass spectrum $\left(U\left(M, M_{2}\right) \approx C M^{u} / 2\right)$ :

$$
J=J(M, t)=2 f_{\text {in }} \int_{0} d M_{2} M_{2} U\left(M, M_{2}\right) f_{2} \rightarrow f_{\text {in }}(M, t) C M^{u} \Pi=\text { Const. }
$$

As for the part of the solution associated with the source $f_{s}$, it is sufficient to consider the source in the form of a $\delta$-function to determine the Schechter slope. According to equation (14), an important feature of the asymptotics of the derived solution for small $a-a_{c r}$ differences is the square root $\sqrt{a-a_{c r}}$ in the denominator. We are interested only in this asymptotics with the substitution $a=a(M, t)$, which, according to equation (5), corresponds to large masses and time instants close to the explosion time, although in this case for $u=2$ and a linear right-hand part of equation (11) we can write the complete solution. In view of $a(M, t)=M^{-1}+C \Pi t$ we have for $t \rightarrow t_{c r} \equiv a_{c r} / C \Pi$ the following formula for $K(a(M, t), t)$ :

$$
K \propto \frac{1}{\sqrt{a(M, t)-a_{c r}}}=\frac{1}{\sqrt{\frac{1}{M}-C \Pi\left(t_{c r}-t\right)}},
$$

\footnotetext{
${ }^{6}$ The number of massive galaxies remains unchanged in the case of massive galaxies merging with low-mass galaxies, which we consider here.
} 
where $t_{c r}$ corresponds to the vertical asymptote of the hyperbola (7) for $a=a_{c r}$. Hence, given fs $f_{s} \propto M^{-u} K(a, t)$ it follows that for sufficiently large masses and sufficiently small $t_{c r}-t$ from (17) the dependence of the solution on mass has a power-law form

$$
f_{s} \propto M^{-3 / 2}
$$

In the general case $u \neq 2$ complex transcendent equations can be derived for the coordinates of the tangent point and intersection points of "hyperbola" (7) with the curves corresponding to the right-hand side of equation (11). However, in the asymptotic domain that is of interest to us, near the multiple root of equation (11) and for small $\delta a \equiv a-a_{c r} \ll a_{c r}$, we again have a quadratic equation. Therefore the above reasoning remains true and the square root of $\delta a$ again appears in the denominator of the formula for $K(a, t)$ (see Appendix 4):

$$
K(a(M, t), t) \propto \frac{1}{\sqrt{a(M, t)-a_{c r}}}=\frac{1}{\sqrt{\left.(u-1)^{-1} M^{-{ }^{u-1}}\right)-C \Pi\left(t_{c r}-t\right)}}
$$

It follows from this that although the position of the tangent point and vertical asymptote of the hyperbola that determines the position of the explosion point depends on all parameters, the MF slope that we compute (the Schechter slope) is determined solely by the dependence of the probability of mergers on mass, i.e., only by its uniformity index $u$

$$
f_{s}=M^{-u} K(a(M, t), t) \propto M^{-\frac{u+1}{2}} .
$$

The uniformity index of the probability of galaxy mergers is known for the two extreme cases [20, 25, 26]. If the masses of merging galaxies are relatively small, then $u=2$. If the masses are sufficiently large, we must take into account the gravitational focusing. In this case, the probability of mergers is proportional to $\propto\left(M_{1}+M_{2}\right)\left(R_{1}+R_{2}\right)$, where $R$ is the characteristic radius of a galaxy.Hence the dependence of the radius of a galaxy on its mass, $R \propto M^{\beta}$, becomes important. The uniformity degree in the domain of large masses is therefore equal to $u=1+\beta$. The Faber-Jackson and Tully-Fisher relations imply $\beta=1 / 2$. (The parameter $\beta$ may differ from $1 / 2$ at large $z$.)

We can thus make the following conclusions concerning the Schechter slope $\alpha$ of the MF. We have $-\alpha=u=2$ in the domain of large $z$, where the 
galaxy masses are small and the result is influenced by the initial MF. This slope coincides with the results of observations at $z=6-8$ (see Fig. 1). The contribution of the source for small masses with $u=2$ becomes important at intermediate $\mathrm{z}$, and we obtain $-\alpha=(u+1) / 2=1.5$. At these $z$ the part of the distribution that is determined by the initial MF is in the domain of sufficiently large masses, where gravitational focusing should manifest itself. This also results in $-\alpha=u=1+\beta=1.5$ and is approximately consistent with observations for $z=3-5$. Finally, at small redshifts, where the source generates the largest masses, $\alpha=(u+1) / 2=1+\beta / 2=1.25$, which is exactly equal to the well-known Schechter exponent at the present epoch.

\section{REGULARIZATION OF THE SOLUTION AND MAXIMUM MASSES}

The solution with the $\delta$-function has an obvious drawback: it is nonexistent (formally becomes infinite) in the case of a multiple root in the argument of the $\delta$-function. Yet it is the multiple root that corresponds to the maximum possible mass at fixed $t$. The formally derived MF then goes to infinity. Hence the MF that decreases in accordance with a power law at large masses goes through a minimum whose position is easy to find in the simplest cases. Thus at $u=2$ the condition for a minimum is satisfied at $M=M_{\min }(t)=$ $\frac{3}{4} M_{\max }(t)$, where $M_{\max }(t)=\frac{1}{C \Pi\left(t_{c r}-t\right)}$ is the "maximum" mass 7 at which $a=a_{c r}$ and the derived MF becomes infinite.

The above means that the solution has to be regularized. Regularization can be achieved by "spreading" the $\delta$-function in one way or another. This procedure takes into account the physically obvious condition that a galaxy cannot instantly separates from cosmological expansion (because of the finite buildup time scale of gravitational instability). This condition can also be formulated in terms of masses assuming that at a given time instant the galaxies that separates from cosmological expansion lie within a small, but finite interval of masses $\Delta \ll M$ near $\bar{M}(t)$. The critical parameter $a_{c r}$ then splits into two parameters $a_{c r} \rightarrow a_{c r}^{ \pm}\left(a_{c r}^{-}>a_{c r}^{+}\right)$corresponding to the tangent points of the hyperbola and the two straight lines $( \pm)$ that bound the right-hand side of equation (11), where (see Appendix)

\footnotetext{
${ }^{7}$ At $t \rightarrow t_{c r}$ this maximum mass becomes infinite itself as a consequence of explosive evolution, where infinite mass is attained in finite time.
} 


$$
a_{c r}^{ \pm}=\frac{u}{u-1}(A C \Pi)^{\frac{u-1}{u}} \mp A C \Pi \frac{\Delta}{2}, \quad x^{ \pm}=A(A C \Pi)^{-1 / u} \mp A \frac{\Delta}{2} .
$$

The regularized solution is finite and can be used to determine the maximum mass $M_{\max }(t)$ of the distribution as a function of time (we restrict this analysis to proper time).

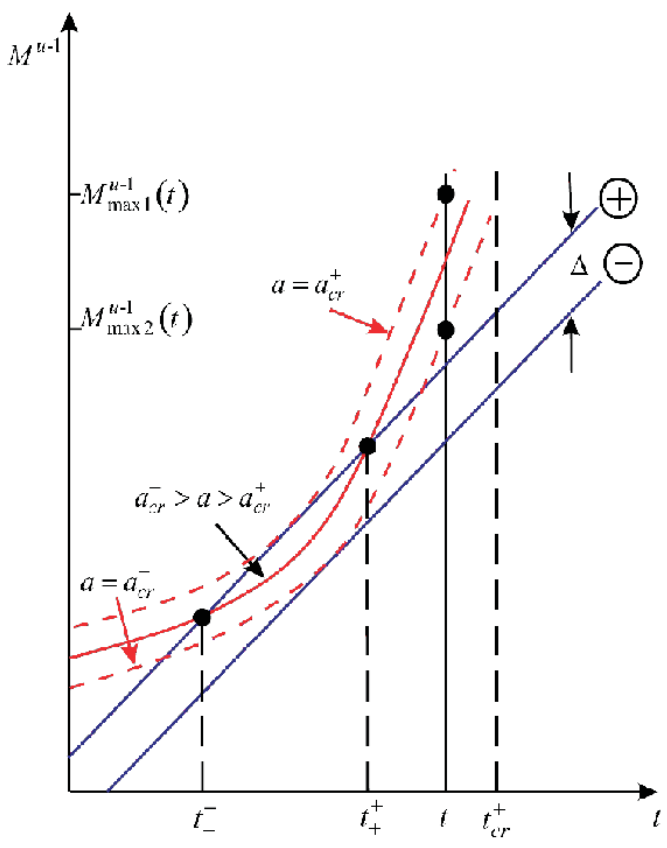

Figure 3: The case of two intersection points of the hyperbola and the $( \pm)$ lines bounding the nonzero source in the kinetic equation at $a_{c r}^{-}>a>a_{c r}^{+}$. The $a=a_{c r}^{ \pm}$values of the integral correspond to the tangent points of the hyperbola and $( \pm)$ lines. The times $t_{ \pm}^{+}$correspond to the intersection of the hyperbola with the upper line $(+)$ at $a>a_{c r}^{-}$. This case does not allow the transition to $\Delta=0$. This mass domain corresponds to the largest MF masses lying in the interval $M_{\max 2}(t)<M<M_{\max 1}(t)$.

Let us assume for simplicity $\Phi(t)=\Phi$. Only those portions where the right-hand side of equation (11) differs from zero contribute to the integral $\mathrm{K}(10)$. The solution of the kinetic equation with a source in the domain of parameters satisfying the condition $a>a_{c r}^{-}>a_{c r}^{+}$has the following form for $t>t_{+}^{+}$(see Appendix 5 and Fig. 3) 


$$
K(a, t)=\frac{2 \Phi \varsigma}{\Delta}\left\{\sqrt{a-a_{c r}^{+}}-\sqrt{a-a_{c r}^{-}}\right\},
$$

where $\varsigma=\sqrt{(-d \mu / d a) /\left(d^{2} \mu / d t^{2}\right)}$ at $a=a_{c r}$. We derive from this the same result at $\Delta \rightarrow 0$ with a feature corresponding to the source in the form of the $\delta$-function:

$$
K(a, t) \rightarrow \Phi \varsigma \frac{A C \Pi}{\sqrt{a-a_{c r}}}
$$

For $\Delta \neq 0$

$$
K(a, t)=\frac{2 \varsigma \Phi}{\Delta} \frac{a_{c r}^{-}-a_{c r}^{+}}{\sqrt{a-a_{c r}^{+}}+\sqrt{a-a_{c r}^{-}}}=\frac{2 \varsigma \Phi \cdot A C \Pi}{\sqrt{a-a_{c r}^{+}}+\sqrt{a-a_{c r}^{-}}} .
$$

For $a \rightarrow a_{c r}^{-}$cr the mass and the corresponding MF value reach their maxima for this domain $t_{c r}^{-}>t>t_{-}^{+}\left(t_{c r}^{ \pm} \equiv a_{c r}^{ \pm} / C \Pi\right)$

$$
K_{\max }=\frac{2 \varsigma \Phi \sqrt{A C \Pi}}{\sqrt{\Delta}}, \quad M_{\max }^{-}(t)=\frac{1}{\left[(u-1) C \Pi \cdot\left(t_{c r}^{-}-t\right)\right]^{\frac{1}{u-1}}} .
$$

Thus in the case of a finite spread $\Delta$ we obtain a finite result for the MF at the point corresponding to the maximum mass for the domain considered. This MF value depends on the spread $\Delta$, which becomes a measurable physical parameter.

Consider now the domain of parameters corresponding to even larger masses, i.e., to the case of two intersections $a_{c r}^{-}>a>a_{c r}^{+}$(see Fig. 4). For $t>t_{+}^{+}$

$$
K=\frac{2 \varsigma \cdot \Phi}{\Delta} \sqrt{a-a_{c r}^{+}} .
$$

The largest mass $M_{\max 1}(t)$ corresponds to the upper boundary $a=a_{c r}^{+}$of the domain where the MF becomes zero. As is evident from equation (26), $\mathrm{K}$ vanishes in accordance with a square-root law. This dependence is superimposed by a power-law decrease due to the relation $f=M^{-u} F(M, t)$. We thus derive a Schechter function analog for the MF, which differs in the decrease law at large masses: the exponential decrease described by equation 
(1) is replaced by the square-root decrease $\sqrt{M_{\max 1}-M}$. Such a behavior, however, is determined by the form of the source localization, which vanishes outside the band $\Delta$. The maximum mass is equal to

$$
M_{\max 1}(t)=\frac{1}{\left[(u-1) C \Pi\left(t_{c r}^{+}-t\right)\right]^{\frac{1}{u-1}}} .
$$

All the above formulas describe explicitly the explosive behavior of the MF. At $t \rightarrow t_{c r}^{+}-0$ the maximum mass goes to infinity.

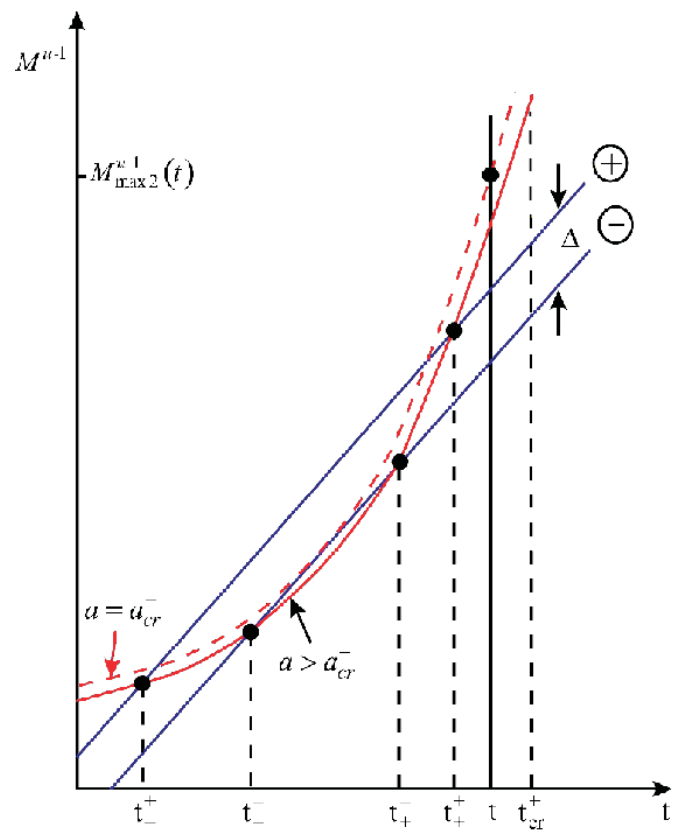

Figure 4: The case of four points of intersection between the hyperbola and the $( \pm)$ lines bounding a nonzero source in the kinetic equation $a>a_{c r}^{-}$. Here $\Delta$ is the mass spread width of the localized source. The value of the integral $a=a_{c r}^{-}$corresponds to the tangent point of the hyperbola and the lower line. The times $t_{ \pm}^{+}$correspond to the intersection of the hyperbola with the upper $(+)$ and lower (-) bounding lines, respectively, at $a>a_{c r}^{-}$. The case considered allows the transition to $\Delta=0$. The position of the maximum mass $M_{\max 2}$ in this mass domain is indicated.

We derived a solution in the form of a modified Schechter function (Fig. 5) with a power-law portion at the "light" end and square-root vanishing at the 
"heavy" end. Unlike the estimated MF slope (Section 6), this result is model dependent. The very presence of a local maximum in the domain of large masses appears to indicate that the spread parameter $\Delta$ is sufficiently small, and requires further investigation. Note that if considered as a function of parameter $a$, the MF decreases as $1 / \sqrt{a}$, making the domain of the smallest possible parameter values that we analyzed above especially important.

\section{CONCLUSIONS}

In this paper we restricted our analysis to the discussion of the slope (the Schechter slope) of the mass function of galaxies, which is most simply related to the physical properties of the problem in terms of the merger model: it is determined solely by the exponent of the dependence of the cross section of galaxy mergers on the mass of the biggest galaxy. The solution obtained confirms that explosive evolution of the MF as a result of minor mergers may, in principle, explain the observed evolution of the low-mass end of the MF up to $z=8$. The computation of the other MF parameters requires invoking a considerably greater amount of astronomical data.8 The most important requirement for the obtained solution is that the time scale of explosive evolution should be shorter than the Hubble time. This criterion can be satisfied only by imposing certain constraints on the masses, radii, and velocities of galaxies with the allowance for the dominating contribution of dark matter [6, 15. In particular, the average density of the mass contained in galaxies (halos) should be more than two orders of magnitude higher than the average density of matter in the Universe. Thus the mergers seem to occur in groups and protoclusters inside larger-scale halos and also in the walls, filaments, and knots of the large-scale structure [27]. The interaction in the process of merging is purely gravitational and we therefore used the known galaxy merger probabilities in our estimates [20, 25, 26]. A proper description of observational data may mean that dark matter behaves as a collisional medium in the process of merging, as it was already pointed out in the literature (see references in [11] ). This may be a result of violent relaxation [28] driven by strong fluctuations of the gravitational field in galaxy mergers.

Note a characteristic difference between the form of the MF derived and

\footnotetext{
${ }^{8}$ Furthermore, in this case we have to face a number of unsolved problems, e.g., the so-called downsizing, where starting from $z \approx 2-3$ the luminosities decrease toward $\mathrm{z}$ $=0$ instead of continuing to increase. A possible solution of this paradox may consist of taking into account the contribution of galactic activity to luminosity.
} 


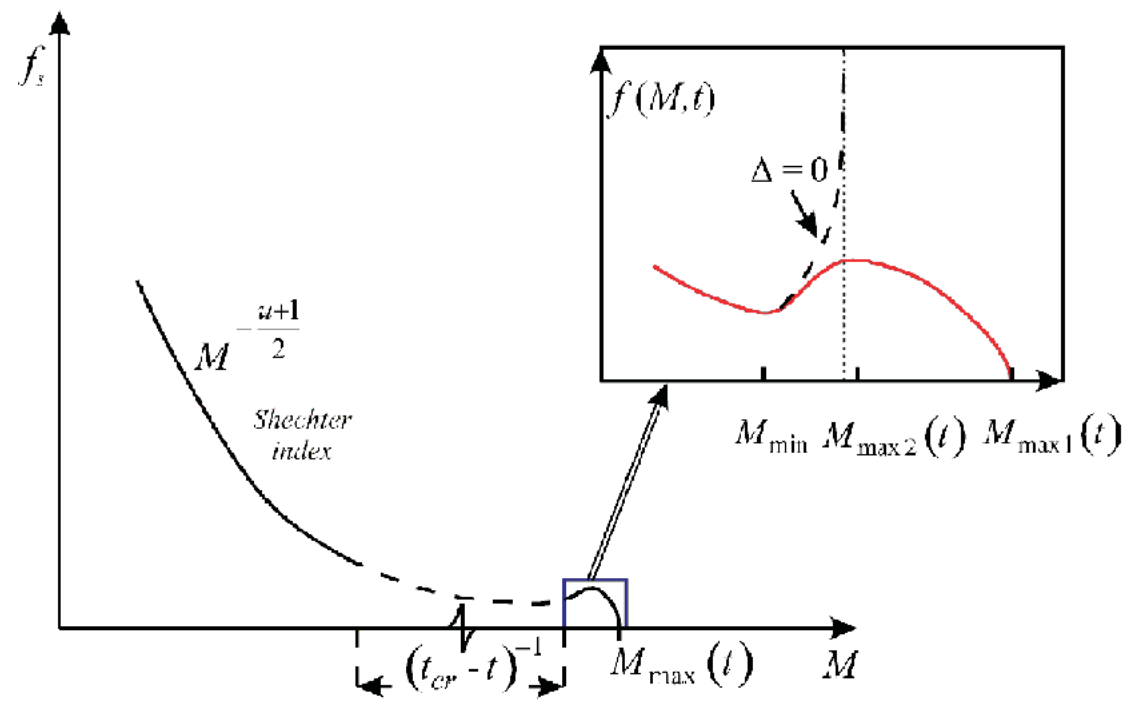

Figure 5: The mass function $f_{s}=M^{-u} K(M, t)$ established as a result of mergers with small mass increments. The dashed line shows the MF singularity in the case of a $\delta$-function source. The MF vanishes at $M=M_{\max }(t)$ because of the adopted stepwise regularization model

the Schechter function: a rising portion of the MF superimposed on the overall decline before the maximum masses (Fig. 5). The parameters of this rising portion are related to the scale lengths of gravitational instability and collapse during the separation of a galaxy from general cosmological expansion.

\section{ACKNOWLEDGMENTS}

We are grateful to the participants of the "Astroparticle Physics" (Kiev, 2011), "Gravitation and Cosmology" (Kiev, 2012), "Electromagnetic Methods of Environmental Studies" (Kharkov, 2012), and "Topical problems of extragalactic astronomy" (Pushchino, 2013) conferences for useful critical comments, and to A. Donets and Yu. Rudnev for their assistance with preparing the figures. This work was supported in part by the "Cosmomicrophysics" program of the National Academy of Sciences of Ukraine. 


\section{APPENDIX}

\section{A1. Derivation of the Differential Kinetic Equation}

The Smoluchowski integral kinetic equation [20]

$$
\frac{\partial}{\partial t} f(M, t)=I_{s t}+\varphi(M, t)
$$

with collision integral

$$
\begin{gathered}
I_{s t}=\iint d M_{1} d M_{2}\left\{W_{M \mid M_{1}, M_{2}} f_{1} f_{2}-W_{M_{1} \mid M_{2}, M} f_{2} f-W_{M_{2} \mid M, M_{1}} f f_{1}\right\} \\
W_{M \mid M_{1}, M_{2}}=U\left(M_{1}, M_{2}\right) \delta\left(M-M_{1}-M_{2}\right) \\
U\left(M_{2}, M_{1}\right)=U\left(M_{1}, M_{2}\right), \quad f=f(M, t), \quad \text { etc. }
\end{gathered}
$$

can be reduced to a differential equation if the kernel function $U\left(M_{1}, M_{2}\right)$ is not singular at $M_{2} \rightarrow 0$, which is the case for galaxy mergers [20, 25]

$$
U\left(M_{1}, M_{2}\right) \rightarrow \frac{C}{2} M_{1}^{u} \quad, \quad M_{1} \gg M_{2},
$$

if the main contribution to $I_{s t}$ is provided by mergers of massive galaxies with low-mass galaxies. In this case the integrand can be expanded into $M_{2} \ll M, M_{1}$ (see [9, 10]) and reduced to the form (2), where $\Pi$ is the total mass of low-mass galaxies. We now rewrite $I_{s t}$ in the form

$$
\begin{gathered}
I_{s t}=2 \int_{0}^{M / 2} d M_{2}\left\{U\left(M-M_{2}, M_{2}\right) f_{2} f\left(M-M_{2}, t\right)-U\left(M_{1}, M_{2}\right) f_{2} f\right\} \\
-2 \int_{M / 2}^{\infty} d M_{2} U\left(M, M_{2}\right) f_{2} f .
\end{gathered}
$$

We then expand the functions containing the difference $M-M_{2}$ into $M_{2} \ll$ $M / 2$ and drop the terms that are small in the ratio of small to large masses to reduce the collision integral to the form $I_{s t} \simeq-\frac{\partial J}{\partial M}$, where $J$ denotes the flux of the number of massive galaxies across the spectrum. Under the assumptions made it can be written in the form

$$
J(M, t)=C M^{u} f(M, t) \Pi(t),
$$




$$
\Pi(t)=\int_{0}^{M / 2} d M_{2} M_{2} f\left(M_{2}, t\right) .
$$

We drop the upper limit, because we assume that the main contribution to $\Pi$ is provided by masses $M_{2} \ll M$. Note that the Smoluchowski equation is derived from the full kinetic equation for the distribution function that depends not only on masses, but also on the coordinates and velocities (momenta) of galaxies via an averaging procedure. Long-range interactions result in faster establishment of velocity distributions compared to mass distributions and therefore the distribution function can be factorized. We now average it over the velocities and, given the linear nature of the equation in the approximation of minor mergers, derive an equation of the form (2), where the coefficient C? appears under the averaging sign. In the case of a sufficiently narrow velocity distribution this results in replacing the velocities with their mean values. Averaging over the coordinates results in the appearance of the mean value with the weight accounting for the large-scale structure, which requires a special analysis.

\section{A2. Probability of Galaxy Mergers}

The coefficient in the mass conservation law in the merger probability $U\left(M_{1}, M_{2}\right) \rightarrow U=\langle\sigma v\rangle$, where the angular brackets, which we hereafter omit, denote averaging over momenta $p, \sigma$ is the merger cross section, and $v=\left|v_{1}-v_{2}\right|$ is the relative velocity of the two galaxies. The cross section is equal to $\sigma=\pi R^{2}\left(1+G M / R v^{2}\right) \varphi$, where $G M / R v^{2}$ is the gravitational focusing parameter [15] [15]; $\varphi=\left(1+R v^{2} / G M\right)^{-\xi}$ is the multiplier that accounts for the dependence of merger probability in the case of a head-on collision on relative velocity. In these formulas we adopt $\xi>0$, $R=R_{1}+R_{2}$, and $M=M_{1}+M_{2}$. At $G M / R v^{2} \gg 1$ the formula for the cross section becomes $\sigma \approx R M \cdot \pi G / v^{2}$. The probability uniformity degree is determined by the parameter $u$, which is determined by expression $U\left(\lambda M_{1}, \lambda M_{2}\right)=\lambda^{u} U\left(M_{1}, M_{2}\right)$. The dependence of the radius on mass, $R_{1} \propto M_{1}^{\beta}$, is determined by the Tully-Fisher and Faber-Jackson laws [1, 11. The cross section may, in the general case, contain dimensionless multipliers depending on the ratios of masses, radii, and velocities, which have no effect on the uniformity degree, but are nevertheless important for the asymptotics. Here we adopt the simplest "elastic" variant [25, 26] for a collisionless velocity distribution, for which 


$$
\begin{gathered}
u=1+\beta, \quad\left(G M / R v^{2} \gg 1\right) ; \quad u=\xi(1-\beta)+2 \beta, \\
\left(G M / R v^{2} \ll 1, \quad \xi \leq 2\right) ; \quad u=2, \quad(\xi \geq 2) .
\end{gathered}
$$

As for the order of magnitude of the probability and, consequently, the estimated time scale of explosive evolution, there still remains a large degree of uncertainty, even without taking into account the so far insufficiently known properties of dark matter. However, we assume that this time scale is shorter than the Hubble time. For this to be true, the condition $\Pi / \rho \geq 10^{2}$, at least, should be satisfied, where $\rho$ is the average matter density in the Universe. According to [3], such a condition is satisfied in the case of halo collapse.

A3. Solving the Kinetic Equation Using the Method of Characteristics

For convenience, we denote the mass that appears in the first integral at time $t=0$ as $M_{0}$. We then have

$$
\begin{gathered}
a\left(M_{0}, t=0\right)=\frac{1}{(u-1) M_{0}^{u-1}} ; M_{0}=\left[\frac{1}{(u-1) a(t=0)}\right]^{\frac{1}{u-1}} ; \\
f_{0}\left(M_{0}\right)=f_{0}\left(\left[\frac{1}{(u-1) a(t=0)}\right]^{\frac{1}{u-1}}\right) .
\end{gathered}
$$

Accordingly, $F(M, t)=M^{u} f(M, t) ; F\left(M_{0}, t=0\right)=M_{0}^{u} f_{0}\left(M_{0}\right)$; whence it follows that

$$
F\left(M_{0}, t=0\right)=\left[\frac{1}{(u-1) a(t=0)}\right]^{\frac{u}{u-1}} f_{0}\left(\left[\frac{1}{(u-1) a(t=0)}\right]^{\frac{1}{u-1}}\right)
$$

It follows from this for arbitrary $M$ and $t$ that

$$
F(M, t)=\left[\frac{1}{(u-1) a(M, t)}\right]^{\frac{u}{u-1}} f_{0}\left(\left[\frac{1}{(u-1) a(M, t)}\right]^{\frac{1}{u-1}}\right) .
$$

As it must be, $F(M, t)$ is a function of integral $a(M, t)$, which satisfies the initial condition. We now substitute $a(M, t)$ in the form 


$$
\frac{1}{(u-1) a(M, t)}=\frac{M^{u-1}}{C \Pi t(u-1) M^{u-1}+1}
$$

and use $f(M, t)=M^{-u} F(M, t)$ to derive formula (9) for $f_{i n}(M, t)$. The first integral $b(M, t)$ of the second equation in (4) for the zero initial condition for this part of the solution results in formula $(9)$ for $f_{s}(M, t)$.

A4. Roots of the Delta-Function Let us now consider the more general case of $u>1, \bar{M}(t)=t^{s} / A, \Pi=$ const. For the above conditions

$$
\tau(x)=C \Pi \cdot x, \quad \mu(a, x)=[(u-1)(a-C \Pi x)]^{\frac{1}{1-u}},
$$

and the equation for the roots of delta-function acquires the following form

$$
\frac{1}{a-C \prod x}=\frac{u-1}{A^{u-1}} x^{(u-1) s} .
$$

The left-hand part of the equation as a function of $x$ is a (generalized) hyperbola $(a>0)$ with the vertical asymptote $x_{a s}=a(C \Pi)^{-1}$, and the right-hand part is a growing power-law function. Equation (A2) may have no real roots (if the hyperbola does not intersect with the power-law function), or have two different roots, or one multiple root in the case if the two curves touch each other. The condition to be satisfied in the latter case is that the two functions and their derivatives should be equal at the tangent point. It is more convenient to use the logarithmic derivative

$$
\frac{1}{a-C \Pi x}=(u-1) s x^{-1} .
$$

We now exclude $a-C \Pi x$ from formulas above to find the tangent point

$$
x_{\text {tan }}^{(u-1) s+1}=\frac{s}{C \Pi} A^{u-1} .
$$

We then substitute $x_{\tan }$ to find the value of parameter $a$ corresponding to the multiple root:

$$
\begin{array}{r}
a=a_{c r}=C \Pi\left[1+\frac{1}{(u-1) s}\right] x_{t a n}= \\
=C \Pi\left[1+\frac{1}{(u-1) s}\right]\left[\frac{s}{C \Pi} A^{u-1}\right]^{\frac{1}{(u-1) s+1}} .
\end{array}
$$


In the $s=1$ case the formulas simplify to

$$
a_{c r}=\frac{u}{u-1}(A C \Pi)^{1-\frac{1}{u}}, \quad x_{t a n}^{u}=\frac{1}{C \Pi} A^{u-1} .
$$

In the simplest case of $u=2$ and $s=1$ they yield

$$
a_{c r}=2 \sqrt{A C \Pi}, \quad x_{t a n}=\sqrt{\frac{A}{C \Pi}} .
$$

Thus for $a>a_{c r}$ we have two roots: $x_{-}<x_{t a n}, x_{+}>x_{t a n}$.

We now return to the general case to obtain, by introducing parameter $\gamma=$ $(u-1) s$,

$$
x_{\text {tan }}^{\gamma+1}=\frac{s}{C \Pi} A^{u-1}, \quad a_{c r}=C \Pi\left[1+\frac{1}{\gamma}\right]\left[\frac{s}{C \Pi} A^{u-1}\right]^{\frac{1}{\gamma+1}} .
$$

The master equation in dimensionless variables $\tilde{a}=a / a_{c r}, \quad T=x / x_{\text {tan }}$ has the form:

$$
\frac{1}{(\gamma+1) \tilde{a}-\gamma T}=T^{\gamma}
$$

Expanding it into small $0<\tilde{a}-1 \ll 1$ and $|\delta T| \ll 1$ in the vicinity of the tangent point yields the quadratic equation $\gamma(\delta T)^{2}-2 \gamma(\tilde{a}-1) \delta T-$ $2(\tilde{a}-1)=0$, roots at $\tilde{a}-1 \ll 1$ are equal to

$$
\delta T_{ \pm} \approx \pm \sqrt{2(\tilde{a}-1) / \gamma} .
$$

A5. Regularization We now replace the $\delta$-function in the right-hand part of the kinetic equation by a step function of finite width $\Delta>0$

$$
\begin{array}{r}
\Phi(M, t)=\delta_{\Pi}(M-\bar{M}(t)) \Phi(t), \\
\delta_{\Pi}(x)=\frac{1}{\Delta}\left[\theta\left(x+\frac{\Delta}{2}\right)-\theta\left(x-\frac{\Delta}{2}\right)\right] \Phi(t),
\end{array}
$$

where $\theta(x)$ is the Heaviside function. $\theta(x)=0$ for $x<0$ and $\theta(x)=1$ for $x \geq 0$. We then have for $K(a, t)$ : 


$$
K(a, t)=\int_{0}^{t} d x \delta_{\Pi}[\mu(a, x)-\bar{M}(x)] \Phi(x) .
$$

The integrand differs from zero if

$$
\bar{M}(x)-\frac{\Delta}{2} \leq \mu(a, x) \leq \bar{M}(x)+\frac{\Delta}{2} .
$$

Each of the two boundary lines $y=\bar{M}(x)+\sigma \frac{\Delta}{2}$ (where $\sigma= \pm$ ) may intersect twice the hyperbola $y=\mu(a, x)$ at $a>a_{c r}^{\sigma}$. The case $a=a_{c r}^{\sigma}$ corresponds to the contact of the curve $\bar{M}(x)+\sigma \Delta / 2$ and hyperbola $\mu(a, t)$ at the points $x=x^{\sigma}$ found from the following conditions:

$$
\begin{gathered}
\mu(a, x)=\bar{M}(x)+\sigma \frac{\Delta}{2} \\
\frac{\partial \mu(a, x)}{\partial x}=\frac{d \bar{M}(x)}{d t} .
\end{gathered}
$$

We find at $\Pi=$ const,$\quad \bar{M}(t)=x / A$,

$$
\begin{array}{r}
\frac{x^{\sigma}}{A}=(A C \Pi)^{-1 / u}-\sigma \frac{\Delta}{2}, \\
a_{c r}^{\sigma}=\frac{u}{u-1}(A C \Pi)^{\frac{u-1}{u}}-\sigma A C \Pi \frac{\Delta}{2}, \quad \sigma= \pm .
\end{array}
$$

Note that $x^{+}<x^{-}$and $a_{c r}^{+}<a_{c r}^{-}$, and $a_{c r}^{-}-a_{c r}^{+}=A C \Pi \cdot \Delta$. We obtain two points of intersection $x_{-}^{+}(a) \leq x_{+}^{+}(a)$ for $a_{c r}^{+}<a<a_{c r}^{-}$(fig.3) and four points of intersection $x_{ \pm}^{ \pm}(a)$ (with both critical lines) for $a_{c r}^{-}<a$ (fig.4). In the latter case we can pass to the limit of $\Delta \rightarrow 0$ to get the $\delta$-function in the righthand part of the kinetic equation:

$$
\sqrt{a-a_{c r}^{\sigma}} \approx \sqrt{a-a_{c r}}\left(1+\sigma \frac{\Delta}{4} \frac{A C \Pi}{\left(a-a_{c r}\right)}\right),
$$

whence it follows that (cf. (22))

$$
\sqrt{a-a_{c r}^{-}}-\sqrt{a-a_{c r}^{+}} \approx \frac{\Delta}{2} \frac{A C \Pi}{\left(a-a_{c r}\right)} .
$$


In the case of small deviations of intersection points from the tangent points we have $\delta x_{ \pm}^{\sigma} \equiv x_{ \pm}^{\sigma}-x^{\sigma}$, where we substituted $x_{ \pm}(a)$ into $a=a_{c r}^{\sigma}$, as follows from equation above, $\delta x_{+}^{\sigma}=-\delta x_{-}^{\sigma}>0$. For $t>x_{+}^{+}$

$$
\begin{aligned}
& K=K^{+} \equiv \frac{1}{\Delta} \int_{t_{-}^{+}}^{t_{+}^{+}} x \Phi(x) \rightarrow \frac{\Phi}{\Delta}\left(t_{+}^{+}-t_{-}^{+}\right) \approx \frac{\Phi}{\Delta}\left(\delta t_{+}^{+}-\delta t_{-}^{+}\right)=\frac{2 \Phi}{\Delta} \delta t_{+}^{+} \\
& \text {At } a_{c r}^{-}<a t>x_{+}^{+} \quad K(a, t)=K^{+}+K^{-}, \text {where } \\
& K^{-}=\frac{\Phi}{\Delta}\left(\delta t_{+}^{-}-\delta t_{-}^{-}\right)=\frac{2 \Phi}{\Delta} \delta t_{+}^{-} .
\end{aligned}
$$

Correspondingly,

$$
\begin{array}{r}
K(a, t)=\frac{\Phi}{\Delta}\left(\delta t_{-}^{+}-\delta t_{+}^{+}+\delta t_{+}^{+}-\delta t_{+}^{-}\right)= \\
=\frac{2 \Phi}{\Delta}\left(\delta t_{-}^{+}+\delta t_{+}^{+}\right)=\frac{2 \Phi \varsigma}{\Delta}\left(\sqrt{a-a_{c r}^{+}}-\sqrt{a-a_{c r}^{-}}\right),
\end{array}
$$

where we took into account that $\delta t_{ \pm}^{\sigma}= \pm \varsigma \sqrt{a-a_{ \pm}^{\sigma}}$ (see 4.2). The derived formulas can be used to construct a regularized MF (fig.5).

\section{References}

[1] S. Veinberg, Cosmology, Oxford UP, 2008; there is a Russian translation: S. Veinberg,, Kosmologiya. .: URSS, Knizhnyi Dom, 2013.

[2] D.S. Gorbunov, V.A. Rubakov, Introduction to the Theory of the Early Universe. Cosmological Perturbations and Inflationary Theory (World Scientific, New Jersey, 2011).

[3] V.N. Lukash and E.V. Mikheeva, Fizicheskaya kosmologiya (Physical cosmology) (Fizmatlit, Moscow, 2010) [in Russian].

[4] R.J. Bouwens, G.D. Illingworth, M. Franx, H. Ford, Ap.J., 670, 928 (2007); Arxiv: 0707.2080.

[5] R.J. Bouwens, G.D. Illingworth, P.A. Oesch, et al, ApJ, 737, 90 (2011); Arxiv: $1006.4360 \mathrm{v} 4$. 
[6] A. Kats \& V. Kontorovich, Vestn. Kiev. Natsion.Univ., Ser. Astron. 48, 16 (2012).

[7] Minor mergings as a driver of galaxy evolution. Special Session in JENAM-2011, 4-8 July 2011, Book of abstracts. St-Petersburg, CAO RAS at Pulkovo, Russia, 185-192 (2011).

[8] V. Kontorovich, Astron.Astrophys.Trans., 5, \#3, 259 (1994).

[9] R.C. Kennicutt, P. Schweizer \& J.E. Barnes. Galaxies: Interactions and Induced Star Formation. Saas-Fee Advance Course, 26, Springer, $404 \mathrm{p}$ (1998).

[10] O.K. Sil'chenko, in Astronomiya: traditsii, nastoyashchee, budushchee (Astronomy: traditions, present, and future), Ed. by V.V. Orlov, V.P. Reshetnikov, and N.Ya. Sotnikova (St. Petersburg State University, St. Petersburg, 2007), p. 63 [in Russian].

[11] R. Ellis \& J. Silk, New frontiers in cosmology and galaxy formation: challenges for the future. In: Structure formation in Astrophysics, Ed. G. Chabrier, Cambridge University Press (2008) p. 133; astro-ph/0712286.

[12] J. Silk, G. Mamon, Research in Astron. Astrophys., 12, No. 8, 917 (2012); arXiv:1207.3080.

[13] V. M. Kontorovich, A. V. Kats, and D. S. Krivitskii, JETP Lett. 55, 1 (1992).

[14] A. Cavaliere, B. Colofrancesco, N. Menci, Astrophys. J.,376, p.L37-41 (1991).

[15] L. D. Landau and E. M. Lifshits, Mechanics, Vol. 1, 3rd ed. (Butterworth-Heinemann, 1976).

[16] A. V. Kats and V. M. Kontorovich, in Abstracts of conf. Electromagnetic Methods of Environmental Studies (Kharkov, 2012); http://ri.kharkov.ua/emes/EMES2012_Thesis.pdf. p.160-162. http://ri.kharkov.ua/emes/EMES2012_Thesis.pdf

[17] C. Steidel, Proceedings of the National Academy of Sciences of the USA, 96, 42321 (1999) 
[18] C. Borys, S. Chapman, M. Donahue, et al., MNRAS, 352, 759 (2004).

[19] A. Berciano Alba, L. Koopmans, M. Garrett, et al., Astron. \& Astrophys., A54, 1 (2010).

[20] A.V. Kats and V.M. Kontorovich, Vopr. Atomn.Nauki i Tekhn. (Problems of Atomic Science and Technology) 68, 137 (2010).

[21] V.V. Stepanov, Kurs differentsialnykh uravnenii (A course in differential equations) (Gos. Izd. Fiz.- Mat. Lit.,Moscow, 1959) [in Russian].

[22] V.M. Kontorovich, Radiofiz. Radioastron. 11, 5 (2006).

[23] V. Voloshchuk, Kineticheskaya teoriya koagulyatsii (Kinetic theory of coagulation) (Gidrometeoizdat, Leningrad, 1984) [in Russian].

[24] M. Ernst, in Proceedings of the Sixth Trieste International Symposium on Fractals in Physics, Trieste, Italy , 1985. Eds. by L. Pietronero nd E. Tosatti (North-Holland, Amsterdam, 1986); There is a Russian translation with editors comments.

[25] D.S. Krivitsky, V.M. Kontorovich, Astron. \& Astrophys., 327, 921 (1997).

[26] G. Mamon, Theory of galaxy dynamics in cluster and groups. In: Theory of galaxies: from the early Universe to the present. ASP conf.ser. 197, 377 (2000).

[27] C.S. Frenk and S.D.M. White, Dark matter and cosmic structure, Annalen der Physik, October 1, 2012; arXiv:1210.0544.

[28] S. White, Violent relaxation in hierarchical clustering. In Proceedings of the 36th Herstmonceux Conference in honour of Professor D. LyndenBell's 60th birthday, "Gravitational Dynamics", eds O. Lahav, E. Terlevich, R. Terlevich, Cambridge, U.K., Aug. 7-11, 1995 (Cambridge University Press, Cambridge, New York, 1996); astro-ph/9602021.

Translation by A.K. Dambis. 